University of Wollongong

Research Online

Faculty of Engineering and Information

Faculty of Engineering and Information

Sciences - Papers: Part A

Sciences

$1-1-2014$

Potassium-uracil/thymine ring cleavage enhancement as studied in electron transfer experiments and theoretical calculations

D Almeida

Universidade Nova de Lisboa

M.-C. Bacchus-Montabonel

Universite de Lyon

F Ferreira Da Silva

Universidade Nova de Lisboa

G Garcia

University of Wollongong

P Limao-Vieira

The Open University

Follow this and additional works at: https://ro.uow.edu.au/eispapers

Part of the Engineering Commons, and the Science and Technology Studies Commons

Research Online is the open access institutional repository for the University of Wollongong. For further information contact the UOW Library: research-pubs@uow.edu.au 


\title{
Potassium-uracil/thymine ring cleavage enhancement as studied in electron transfer experiments and theoretical calculations
}

\author{
Abstract \\ We report experimental and theoretical studies on ring cleavage enhancement in collisions of potassium \\ atoms with uracil/thymine in order to further increase the understanding of the complex mechanisms \\ yielding such fragmentation pathways. In these electron transfer processes time-of-flight (TOF) negative \\ ion mass spectra were obtained in the collision energy range $13.5-23.0 \mathrm{eV}$. We note that $\mathrm{CNO}-$ is the \\ major ring breaking anion formed and its threshold formation is discussed within the collision energy \\ range studied. Such decomposition process is supported by the first theoretical calculations in order to \\ clarify how DNA/RNA pyrimidine bases fragmentation is enhanced in electron transfer processes yielding \\ ion-pair formation.

\section{Keywords} \\ electron, studied, enhancement, cleavage, transfer, ring, potassium, thymine, experiments, theoretical, \\ calculations, uracil

\section{Disciplines} \\ Engineering | Science and Technology Studies

\section{Publication Details} \\ Almeida, D., Bacchus-Montabonel, M., da Silva, F. Ferreira., García, G. \& Limão-Vieira, P. (2014). Potassium- \\ uracil/thymine ring cleavage enhancement as studied in electron transfer experiments and theoretical \\ calculations. The Journal of Physical Chemistry Part A: Molecules, Spectroscopy, Kinetics, Environment \\ and General Theory, 118 (33), 6547-6552.
}




\title{
Potassium-Uracil/Thymine Ring Cleavage Enhancement as Studied in Electron Transfer Experiments and Theoretical Calculations
}

\author{
D. Almeida ${ }^{1}$, M.-C. Bacchus-Montabonel ${ }^{2, *}$, F. Ferreira da Silva ${ }^{1}$, \\ G. García ${ }^{3,4}$ and P. Limão-Vieira ${ }^{1,5, *}$ \\ ${ }^{1}$ Laboratório de Colisões Atómicas e Moleculares, CEFITEC, Departamento de Física, Faculdade de \\ Ciências e Tecnologia, Universidade Nova de Lisboa, 2829-516 Caparica, Portugal \\ 2 Institut Lumière Matière, UMR5306 Université Lyon 1-CNRS, Université de Lyon, 69622 \\ Villeurbanne Cedex, France \\ ${ }^{3}$ Instituto de Física Fundamental, Consejo Superior de Investigaciones Científicas, Serrano 113-bis, \\ 28006 Madrid, Spain \\ ${ }^{4}$ University of Wollongong, Centre of Medical Radiation Physics, Wollongong, NSW 2522, Australia \\ ${ }^{5}$ Centre of Earth, Planetary, Space and Astronomy Research, Department of Physics and Astronomy, \\ The Open University, Walton Hall, Milton Keynes, MK7 6AA, UK
}

\begin{abstract}
We report experimental and theoretical studies on ring cleavage enhancement in collisions of potassium atoms with uracil/thymine in order to further increase the understanding of the complex mechanisms yielding such fragmentation pathways. In these electron transfer processes time-of-flight (TOF) negative ion mass spectra were obtained in the collision energy range $13.5-23.0 \mathrm{eV}$. We note that $\mathrm{CNO}^{-}$is the major ring breaking anion formed and its threshold formation is discussed within the collision energy range studied. Such decomposition process is supported by the first theoretical calculations in order to clarify how DNA/RNA pyrimidine bases fragmentation is enhanced in electron transfer processes yielding ion-pair formation.
\end{abstract}

Keywords: $\mathrm{CNO}$ anion, theoretical calculations, negative ion, atomic collisions.

\footnotetext{
* Corresponding authors: Tel: (+351) 2129478 59; Tel: (+33) 472431083

Email addresses:plimaovieira@fct.unl.pt (P. Limão-Vieira); bacchus@univ-lyon1.fr (M-C Bacchus-Montabonel)
} 


\section{Introduction}

Radiation-induced damage to biologically relevant molecular systems has recently come under great scrutiny by the international scientific community due to recent findings highlighting the lethality of low-energy electrons as a DNA/RNA damaging agent ${ }^{1}$. However, much still remains to be unveiled regarding the exact molecular mechanisms that occur in the nascent stages of DNA/RNA damage by the incident radiation, in particular the role of third bodies in electron capture processes by the double-helix molecular constituents. Indeed, in such radiation-induced processes, secondary electrons are produced from the ionisation events as well as from their release in the physiological medium from solvated and/or pre-solvated states $^{2,3}$. Though, studying the damaging role of "bound" electrons to DNA/RNA may provide another route to better understand the nature of such processes which seem to be more attuned to the physiological environment than simple free electrons. In particular, studying electron transfer processes from donating atoms with biologically relevant molecules can provide valuable information on the role of such "bound" electrons ${ }^{4-8}$.

A quite extensive set of theoretical ${ }^{2,7,9-14}$ and experimental ${ }^{7,9-11,14-21}$ dissociative electron attachment (DEA) studies to pyrimidine nucleobases have gradually been published over the last few years. In the particular case of uracil/thymine, these DEA studies report the dehydrogenated parent anion formation as the dominant fragmentation pathway, ${ }^{2,9,22-24}$ which, together with theoretical calculations, has been extensively studied and reported to derive exclusively from hydrogen abstraction from the N1 and N3 sites ${ }^{9,15,18,20}$. However, for potassium collisions with such molecular targets, the results are significantly different. Several studies on the negative ion formation in potassium-molecule collisions have been performed $^{5,7,11,25-27}$ and, although the dehydrogenated parent anion is also one of the main fragments, the most intense fragment anion has been assigned to $\mathrm{CNO}^{-}$, which necessarily requires ring breaking and hence, access to very different anionic states to those that result in dehydrogenation from the $\mathrm{N}$-sites ${ }^{15,25}$. In particular, a recent study on the metastable decay of methylated pyrimidine derivatives (temporary negative) ions resulting in $\mathrm{CNO}^{-}$formation upon electron capture/transfer, have shown the relevance of hydrogen abstraction (either from $\mathrm{N} 1$ or $\mathrm{N} 3)^{11}$ in the site and bond selectivity decomposition mechanism. However, from a literature survey we note that theoretical descriptions of intramolecular decomposition processes are scarce or even absent within the context of electron transfer. As such there has been an increased need to perform more comprehensive and detailed studies on how some of the most dominant fragments, a part from the dehydrogenated parent anion, are formed. Furthermore, the present electron transfer studies show that other fragments, as is the case of 
$\mathrm{CNO}^{-}$, are more relevant within the pyrimidine's decomposition (than the dehydrogenated parent anion) which may compromise the DNA/RNA integrity after electron capture.

As such, the aim of this contribution is to make use of the information supplied by the present theoretical calculations and together with the experimental data (mainly on thresholds), to construct a clearer picture of the dissociation mechanisms in the pyrimidinic ring after electron transfer, with a special emphasis on the pathway resulting in $\mathrm{CNO}^{-}$ formation. We report a set of data that shows the threshold of formation for the most intense fragment anion $\left(\mathrm{CNO}^{-}\right)$in electron transfer experiments stemming from an initial electron capture into high-energy anionic states $(>5 \mathrm{eV})$ as proposed in previous studies ${ }^{4}$. Using the present theoretical calculations, which take into consideration the presence of the electron donating projectile, we aim at gaining a better description of the electron transfer process in potassium collisions with uracil/thymine. As so, the information gained on the electronic states available in the K-Uracil and K-Thymine allows understanding the reaction pathways resulting in ring breaking.

In the next section, a short discussion on the experimental system used to obtain the TOF mass spectra is presented, followed in section 3 by a discussion on the theoretical method. In section 4 we present experimental results and discussion together with the provided theoretical calculations. Finally, some general conclusions are drawn in section 5.

\section{Experimental details}

The experimental setup used to obtain the present negative ions TOF mass spectra has been described in detail elswhere $e^{5,7,8,11,25-28}$, and so a brief description is given here. Briefly, a neutral hyperthermal potassium beam crosses an effusive molecular beam, and the anionic products are extracted into a TOF mass spectrometer. Potassium cations are produced in an ionic source and accelerated by an electric potential to the entrance of a charge exchange chamber containing potassium vapour. The incoming hyperthermal potassium cations resonantly charge exchange in collisions with neutral thermal potassium atoms, resulting in a neutral hyperthermal potassium beam. A set of deflecting plates outside the oven ensures the removal of the remaining potassium cations in the beam. In the present measurements, the neutral potassium energy is varied between 13.5 and $\sim 23.0 \mathrm{eV}$ in the laboratory frame. Uracil and thymine were heated to $400 \mathrm{~K}$ and careful procedures were taken in order to avoid thermal decomposition (for further details see reference ${ }^{20}$ ). The TOF mass spectrometer is of a Wiley-McClaren type operating with a $\sim 1 \mu$ s width pulsed extraction voltage of $\sim 242$ $\mathrm{Vcm}^{-1}$. The mass resolution is $\mathrm{m} / \Delta \mathrm{m}=125$. Anions are detected by a channeltron with an 
active area of $1 \mathrm{~cm}$ diameter. Systematic background measurements enabled residual gas contributions to be subtracted from the sample spectra. The samples used in the present experiment were purchased from Sigma-Aldrich with a minimum purity of $\geq 99 \%$.

\section{Theoretical method}

The charge transfer reaction between the potassium atom and uracil/thymine nucleobases to form $\mathrm{K}^{+}$and the nucleobases anions is described as the evolution of a quasi-molecular system formed by the potassium atom projectile and the biomolecular target. A model for such collision systems may be developed in the one-dimension reaction coordinate approximation as previously applied for ion-biomolecule collision systems ${ }^{29-31}$. The atombiomolecule collision system is thus treated as a pseudo-diatomic molecule which evolves along the reaction coordinate associated to the distance between the centre-of-mass of the biomolecule and the projectile atom ${ }^{32-34}$. Such a simple approach does not take into account the internal motions of the biomolecule but it has been shown to be acceptable for very fast collision processes where nuclear vibration and rotation motions are much slower than the collision time.

For pyrimidine nucleobases uracil and thymine, the geometry is constructed around a 6membered planar ring ${ }^{29-31}$, considering a perpendicular approach of the potassium atom as this orientation has been clearly shown to be favoured for uracil and thymine targets in collisions with carbon ions ${ }^{35,36}$. The quantum chemical calculations have been carried out by means of the MOLPRO code ${ }^{37}$. The ECP18sdf core-electron pseudopotential has been used to take account of the 18 core electrons of potassium ${ }^{38}$, with the corresponding basis set. The 6$311 \mathrm{G}^{* *}$ basis set has been used for other atoms, taking account of all electrons; calculations have been performed in Cartesian coordinates with no symmetries. All biomolecular targets are considered in their ground state geometry and kept frozen during the collision process. The geometry of the ground state of thymine and uracil has been optimized at the Complete Active Space Self Consistent Field (CASSCF) level of theory and with Density Functional Theory (DFT) approaches ${ }^{35}$. The natural molecular orbitals for the K-Uracil and K-Thymine molecular systems have been determined by state-averaged CASSCF calculations for the reaction coordinate $R=6 \AA$ corresponding to the asymptotic region. Similar $(5,11)$ active spaces corresponding to 5 electrons in 11 orbitals have been considered for both targets in order to compare each system at the same level of accuracy. They are constructed mainly on the $2 \mathrm{p}_{\mathrm{z}}$ and $2 \mathrm{p}_{\mathrm{xy}}$ orbitals centred on oxygen $\mathrm{O} 4$, on the $\pi_{\mathrm{C} 4 \mathrm{O} 4}$ orbital and on the $2 \mathrm{p}_{\mathrm{z}}$ orbitals on C5 and C6 carbon atoms (describing the $\pi_{\mathrm{C} 5 \mathrm{C} 6}$ molecular orbital) with, of course, the $4 \mathrm{~s}$ and 
$4 \mathrm{p}$ orbitals on potassium. The $1 \mathrm{~s}$ orbitals of carbon, nitrogen and oxygen are treated as frozen core. The lowest unoccupied molecular orbitals (LUMOs) for uracil and thymine are shown in Figures 1 and 2, respectively. In order to compare the present results to the LUMOs involved in DEA processes, similar calculations of the neutral ground state of uracil and thymine molecules have been performed and indicated in both figures, respectively. The natural orbitals have been determined as previously at the CASSCF level of theory with $(4,8)$ active spaces involving 4 closed shell electrons of the nucleobases. The corresponding LUMOS are also shown in Figures 1 and 2.

\section{Results and Discussion}

In this section, qualitative and quantitative analyses of the TOF mass spectra yielding $\mathrm{CNO}^{-}(\mathrm{m} / \mathrm{z}=42)$ formation (see Figure 3$)$ obtained at different collisions of potassium atoms with uracil/thymine in the laboratory frame are presented. Such energy range (13.5-22.8 eV) allows discussion and considerations on the intermediate molecular mechanisms supported by the current theoretical results. While most of the discussion is centred on the uracil underlying mechanisms, most of the rationales also apply to thymine given their molecular similarities. Since we are particularly interested on the pyrimidines ring cleavage enhancement yielding the most intense anion, $\mathrm{CNO}^{-}$, the TOF mass data in Figure 3 is for uracil only, although in the case of thymine we observe the same behaviour. Of relevance the fact that uracil and thymine differ on $14 \mathrm{~m} / \mathrm{z}$, which in terms of the potassium-molecule centre-of-mass collision system results in less than $10 \%$ difference in the collision energy.

\subsection{Uracil}

A section of the total TOF negative ions mass spectra of potassium atoms with uracil in an energy collision range from $13.5-22.8 \mathrm{eV}$ is presented in Figure 3 for $\mathrm{m} / \mathrm{z}=42$ formation. This fragment is the dominant product anion for higher collision energies (typically above $30 \mathrm{eV}$ ) whereas is absent below its threshold formation $(<15 \mathrm{eV})$. It is worth noting that the dehydrogenated parent anion (not shown here) is formed in the present collision energy range (for a complete discussion see reference ${ }^{20}$ ), meaning that its threshold of formation is below $13.5 \mathrm{eV}$ (4.6 eV available energy - see Table 1). This is in agreement with DEA resonances observed at $1.1 \mathrm{eV}$ and $1.8 \mathrm{eV}$ and attributed to N1-H and N3-H excision, respectively ${ }^{9}$. However, $\mathrm{CNO}^{-}$formation requires different and concerted mechanisms in the temporary negative ions, $\left(\mathrm{U}^{-}\right)^{\#} /\left(\mathrm{T}^{-}\right)^{\#}$, prior to dissociation. As far as DEA is concerned, $\mathrm{CNO}^{-}$formation is known to result from initial access to a set of high-energy 
anionic states at $\sim 5 \mathrm{eV}, \sim 7 \mathrm{eV}$ and $\sim 9 \mathrm{eV}^{15,20}$. A theoretical electron scattering study by Gianturco et $a l^{12,13}$ on electron attachment to uracil pointed out the presence of several highenergy $\pi^{*}$ and $\sigma^{*}$ states within the ring framework, where an initial access to one of the $\pi^{*}$ states and subsequent intramolecular electron transfer into one of the highly anti-bonding $\sigma^{*}$ states was suggested to be an effective ring-breaking pathway. This rationale was later adapted within the context of electron transfer to thymine and uracil ${ }^{25}$, where the presence of the potassium cation post-electron transfer may greatly enhance formation of such fragment $\operatorname{anion}^{25}$. Briefly, the aforementioned theoretical study ${ }^{12,13}$ calculated the resonance widths of the $\pi^{*}$ and $\sigma^{*}$ states, whose values imply very low auto-detachment lifetimes, thereby not allowing the fragmentation pathway to evolve. However, for the case of electron transfer studies $^{25}$, the presence of the potassium cation post-collision would suppress auto-detachment long enough for the fragmentation pathway successful competition. While this picture seems to adequately describe the intramolecular dynamics, the calculations were performed for a free-electron scattering process, which disregards the presence of a third body $\left(\mathrm{K}^{+}\right.$postcollision).

The theoretical calculations presented in this study attempt to address some of these issues, particularly that regarding the perturbation undergone by the molecule's LUMOs in the presence of the potassium atom. In Figures 1 and 2, the set of calculated orbitals for potassium collisions with uracil and thymine are presented, respectively. As can be seen, while some of the orbitals are somewhat similar in shape to those calculated in other theoretical studies ${ }^{9,12,13}$, there are others that are absent in the aforementioned DEA studies. In particular, the $\sigma_{1} *$ orbitals calculated for thymine and uracil, which represent highly antibonding characters in the $\mathrm{C} 4-\mathrm{C} 5$ and $\mathrm{C} 4-\mathrm{O} 2$ bonds, respectively, are not obtained in the most recent electron scattering calculations ${ }^{9,12,13}$. Furthermore, the calculated $\pi^{*}$ orbitals show significantly different energies and spin densities in comparison with the corresponding LUMOs calculated with electron scattering methods (see ref. ${ }^{12}$ ).

The threshold for $\mathrm{CNO}^{-}$formation lies between 14.5 and $15.5 \mathrm{eV}$ collision energy (5.2 and $5.9 \mathrm{eV}$ available energy in the centre-of-mass frame, see also Table 1) according to the TOF experimental data in Figure 3. Furthermore, a more careful analysis of the high-energy $\left(>17.5 \mathrm{eV}\right.$ ) spectra in Figure 3 shows that a major increase in the $\mathrm{CNO}^{-}$relative yield is observed, which may indicate the access to another high-energy state at approximately 9-10 $\mathrm{eV}$. This analysis is consistent with DEA resonance profiles showing that $\mathrm{CNO}^{-}$is formed through a set of multiple high-energy resonances ${ }^{15,23}$. As such, we can safely attribute $\mathrm{CNO}^{-}$ 
formation through access to a resonance with a threshold at $\sim 5 \mathrm{eV}$ and a second resonance at approximately $9 \mathrm{eV}$.

At this point, in order to better understand the underlying mechanisms and accessed states that are responsible for $\mathrm{CNO}^{-}$formation, one can resort to the provided theoretical calculations. In Figure 1 for uracil, we can identify two $\pi^{*}$ resonances: $\pi_{2}^{*}$ and $\pi_{3} *$ at 5.1 and $10.7 \mathrm{eV}$, respectively, whereas in Figure 2 for thymine we identify a $\pi_{2}^{*}$ at $5.2 \mathrm{eV}$ and a $\pi_{3}^{*}$ at $8.3 \mathrm{eV}$. For the case of thymine, at even higher energies, an $\sigma^{*}$ resonance along the ring coordinates is also present. As discussed in the previous section, given the inaccuracy associated with the calculation of higher-energy states, despite being present, no further $\sigma^{*}$ states are shown. Within this context, it is known that the anti-bonding character associated with the occupation of a $\pi^{*}$ state does not lead directly to bond breaking, however, an intramolecular electron transfer from the initially occupied $\pi^{*}$ to an overlapping $\sigma^{*}$ will most likely result in bond breaking, as long as the nuclear wavepacket in the $\pi^{*}$ state survives long enough to diabatically couple with the repulsive $\sigma^{*}$. As such, we therefore assign the threshold obtained at $\sim 5 \mathrm{eV}$ (14.5 eV collision energy) to an electron transfer to the $\pi_{2}{ }^{*}$ orbital and the aforementioned relative yield increases due to an electron transfer to the $\pi_{3}{ }^{*}$ state. Furthermore, since bond-breaking can only occur through access of $\sigma^{*}$ states, an electron transfer from the $\pi^{*}$ to the $\sigma^{*}$ states is also necessary.

Indeed, a similar mechanism has been proposed in an electron scattering study in uracil $^{12,13}$, where it was shown that occupation of a $\pi^{*}$ state could be an intermediate to access a highly anti-bonding localized $\sigma^{*}$ state that will result in ring (bonds) breaking. However, in these studies ${ }^{12,13}$, it was calculated that the resonance widths of these states are quite wide and hence, this fragmentation pathway would have to compete with auto-detachment ${ }^{12,13}$. At this point, the importance of the potassium cation post-electron transfer is decisive. Indeed, it has been shown in several studies with other molecules that formation of the temporary $\left(\mathrm{K}^{+}+\mathrm{M}^{-}\right)$ (where $\mathrm{K}$ is the potassium atom and $\mathrm{M}$ is the electron-acceptor molecule) coulombic complex appears to prevent detachment of the electron from the electron acceptor, thereby allowing the fragmentation to successfully compete with auto-detachment.

\subsection{Thymine}

Although no experimental measurements similar to those obtained for uracil are shown here, a similar rationale for the discussion on uracil allow us to say that: 1) the molecular geometries of both molecules are very similar; 2) DEA studies on thymine show a $\mathrm{CNO}^{-}$formation resonance profile very similar as in uracil ${ }^{18}$; 3) recent studies on 1-methyl- 
thymine ${ }^{11}$ show a relative increase in $\mathrm{CNO}^{-}$formation very similar to that observed in Figure 3. All of these considerations provide ample support to the assumption that the underlying mechanisms yielding $\mathrm{CNO}^{-}$are similar to those presented for uracil. Regarding the theoretical calculations, Figure 2 shows the set of calculated $\pi^{*}$ and $\sigma^{*}$ orbitals for thymine. Indeed, the general shape of the $\pi^{*}$ states for thymine are roughly similar to their uracil analogues. This is not surprising, given the arguments presented before. As such, the rationale that was constructed for uracil should also be applicable to thymine.

\section{Conclusions}

The present work provides closer insight into the molecular mechanisms underlying $\mathrm{CNO}^{-}$formation, the dominant fragment from electron transfer experiments in collisions of potassium atoms with uracil and thymine. The set of novel theoretical calculations presented in this work account for the presence of the electron donor (potassium atom) and how it affects the LUMOs of uracil and thymine. Such information on the electronic structure has been compared to previous theoretical approach in the context of DEA albeit with significant differences. Additionally, we show for the first time TOF negative ion mass spectra for nearthreshold energies of $\mathrm{CNO}^{-}$formation. Indeed, we show that initial electron occupation of $\pi_{2}$ * and $\pi_{3}{ }^{*}$ resonances (Figures 1 and 2), followed by intramolecular electron transfer to ringbond $\sigma^{*}$ sates, may lead to $\mathrm{m} / \mathrm{z}=42$ anion formation. While the present calculations do neither encompass the full extent of the presence of the electron-donating projectile nor of the collision dynamics, some initial aspects of the electronic states involved in the electron transfer process are indeed highlighted in this work.

\section{Acknowledgements}

DA and FFS acknowledge the Portuguese Foundation for Science and Technology (FCT-MEC) for post-doctoral grants SFRH/BPD/68979/2010 and SFRH/BPD/99261/2013, respectively, and together with PL-V the research grants PEst-OE/FIS/UI0068/2014 and PTDC/FIS-ATO/1832/2012. GG acknowledges partial financial support from the Spanish Ministerio de Economia y Competitividad (Project No. FIS2012-31230). PL-V acknowledges his visiting Professor position at The Open University, UK. MCB acknowledges support from the HPC resources of CCRT/CINES/IDRIS under the allocations 2014-[i2014081566] made by GENCI [Grand Equipement National de Calcul Intensif]. Some of this work forms part of the EU/ESF COST Actions Nano-IBCT-MP1002, CELINA-CM1301 and XLIC-CM1204. 


\section{References}

(1) Boudaïffa, B.; Cloutier, P.; Hunting, D.; Huels, M. A.; Sanche, L. Resonant Formation of DNA Strand Breaks by Low-Energy ( 3 to $20 \mathrm{eV}$ ) Electrons. Science 2000, 287, 1999-2001.

(2) Alizadeh, E.; Sanche, L. Precursors of Solvated Electrons in Radiobiological Physics and Chemistry. Chem. Rev. 2012, 112, 5578-5602.

(3) Sanche, L. Biological Chemistry: Beyond Radical Thinking. Nature 2009, 461, 358.

(4) Almeida, D.; Antunes, R.; Martins, G.; Eden, S.; Ferreira da Silva, F.; Nunes, Y.; Garcia, G.; Limão-Vieira, P. Electron Transfer-Induced Fragmentation of Thymine and Uracil in Atom-Molecule Collisions. Phys. Chem. Chem. Phys. 2011, 13, 1565715665 .

(5) Almeida, D.; Ferreira da Silva, F.; García, G.; Limão-Vieira, P. Selective Bond Cleavage in Potassium Collisions with Pyrimidine Bases of DNA. Phys. Rev. Lett. 2013, 110, 023201.

(6) Almeida, D.; Ferreira da Silva, F.; García, G.; Limão-Vieira, P. Dynamic of Negative Ions in Potassium-D-Ribose Collisions. J. Chem. Phys. 2013, 139, 114304.

(7) Almeida, D.; Kinzel, D.; Ferreira da Silva, F.; Puschnigg, B.; Gschliesser, D.; Scheier, P.; Denifl, S.; García, G.; González, L.; Limão-Vieira, P. N-Site de-Methylation in Pyrimidine Bases as Studied by Low Energy Electrons and Ab Initio Calculations. Phys. Chem. Chem. Phys. 2013, 15, 11431-11440.

(8) Almeida, D.; Ferreira da Silva, F.; Eden, S.; García, G.; Limão-Vieira, P. New Fragmentation Pathways in K-THF Collisions as Studied by Electron-Transfer Experiments: Negative Ion Formation. J. Phys. Chem. A 2014, 118, 690-696.

(9) Burrow, P. D.; Gallup, G. A.; Scheer, A. M.; Denifl, S.; Ptasińska, S.; Märk, T.; Scheier, P. Vibrational Feshbach Resonances in Uracil and Thymine. J. Chem. Phys. 2006, 124, 124310.

(10) Cheng, H.-Y.; Chen, C.-W. Energy and Lifetime of Temporary Anion States of Uracil by Stabilization Method. J. Phys. Chem. A 2011, 115, 10113-10121.

(11) Ferreira da Silva, F.; Matias, C.; Almeida, D.; García, G.; Ingólfsson, O.; Flosadóttir, H. D.; Ómarsson, B.; Ptasińska, S.; Puschnigg, B.; Scheier, P.; et al. NCO(-), a Key Fragment upon Dissociative Electron Attachment and Electron Transfer to Pyrimidine Bases: Site Selectivity for a Slow Decay Process. J. Am. Soc. Mass Spectrom. 2013, 24, 1787-1797.

(12) Gianturco, F. A.; Sebastianelli, F.; Lucchese, R. R.; Baccarelli, I.; Sanna, N. RingBreaking Electron Attachment to Uracil: Following Bond Dissociations via Evolving Resonances. J. Chem. Phys. 2008, 128, 174302. 
(13) Gianturco, F.; Lucchese, R. R. Radiation Damage of Biosystems Mediated by Secondary Electrons: Resonant Precursors for Uracil Molecules. J. Chem. Phys. 2004, $120,7446-7455$.

(14) Scheer, A. M.; Aflatooni, K.; Gallup, G.; Burrow, P. Bond Breaking and Temporary Anion States in Uracil and Halouracils: Implications for the DNA Bases. Phys. Rev. Lett. 2004, 92, 3-6.

(15) Denifl, S.; Ptasińska, S.; Hanel, G.; Gstir, B.; Probst, M.; Scheier, P.; Märk, T. D. Electron Attachment to Gas-Phase Uracil. J. Chem. Phys. 2004, 120, 6557-6565.

(16) Ptasińska, S.; Denifl, S.; Grill, V.; Märk, T. D.; Scheier, P.; Gohlke, S.; Huels, M. A.; Illenberger, E. Bond-Selective H- Ion Abstraction from Thymine. Angew. Chem., Int. Ed. Engl. 2005, 44, 1647-1650.

(17) Ptasińska, S.; Denifl, S.; Grill, V.; Märk, T. D.; Illenberger, E.; Scheier, P. Bond- and Site-Selective Loss of H- from Pyrimidine Bases. Phys. Rev. Lett. 2005, 95, 1-4.

(18) Ptasińska, S.; Denifl, S.; Scheier, P.; Illenberger, E.; Märk, T. D. Bond- and SiteSelective Loss of H Atoms from Nucleobases by Very-Low-Energy Electrons $(<3 \mathrm{eV})$. Angew. Chem., Int. Ed. Engl. 2005, 44, 6941-6943.

(19) Martin, F.; Burrow, P.; Cai, Z.; Cloutier, P.; Hunting, D.; Sanche, L. DNA Strand Breaks Induced by 0-4 eV Electrons: The Role of Shape Resonances. Phys. Rev. Lett. 2004, 93, 6-9.

(20) Hanel, G.; Gstir, B.; Denifl, S.; Scheier, P.; Probst, M.; Farizon, B.; Farizon, M.; Illenberger, E.; Märk, T. Electron Attachment to Uracil: Effective Destruction at Subexcitation Energies. Phys. Rev. Lett. 2003, 90, 188104.

(21) Abdoul-Carime, H.; Gohlke, S.; Fischbach, E.; Scheike, J.; Illenberger, E. Thymine Excision from DNA by Subexcitation Electrons. Chem. Phys. Lett. 2004, 387, 267270.

(22) Denifl, S.; Ptasińska, S.; Hanel, G.; Gstir, B.; Probst, M.; Scheier, P.; Märk, T. D. Electron Attachment to Gas-Phase Uracil. J. Chem. Phys. 2004, 120, 6557-6565.

(23) Denifl, S.; Ptasińska, S.; Probst, M.; Hrusak, J.; Scheier, P.; Märk, T. D. Electron Attachment to the Gas-Phase DNA Bases Cytosine and Thymine. J. Phys. Chem. A 2004, 108, 6562-6569.

(24) Smyth, M.; Kohanoff, J. Excess Electron Localization in Solvated DNA Bases. Phys. Rev. Lett. 2011, 106, 238108.

(25) Almeida, D.; Antunes, R.; Martins, G.; Eden, S.; Ferreira da Silva, F.; Nunes, Y.; Garcia, G.; Limão-Vieira, P. Electron Transfer-Induced Fragmentation of Thymine and Uracil in Atom-Molecule Collisions. Phys. Chem. Chem. Phys. 2011, 13, 1565715665. 
(26) Antunes, R.; Almeida, D.; Martins, G.; Mason, N. J.; Garcia, G.; Maneira, M. J. P.; Nunes, Y.; Limão-Vieira, P. Negative Ion Formation in Potassium-Nitromethane Collisions. Phys. Chem. Chem. Phys. 2010, 12, 12513-12519.

(27) Ferreira da Silva, F.; Almeida, D.; Antunes, R.; Martins, G.; Nunes, Y.; Eden, S.; Garcia, G.; Limão-Vieira, P. Electron Transfer Processes in Potassium Collisions with 5-Fluorouracil and 5-Chlorouracil. Phys. Chem. Chem. Phys. 2011, 13, 21621-21629.

(28) Almeida, D.; Ferreira da Silva, F.; García, G.; Limão-Vieira, P. Dynamic of Negative Ions in Potassium-D-Ribose Collisions. J. Chem. Phys. 2013, 139, 114304.

(29) Bacchus-Montabonel, M.C.; Łabuda, M.; Tergiman, Y.S.; Sienkiewicz, J.E. Theoretical Treatment of Charge-Transfer Processes Induced by Collision of $\mathrm{C}^{\mathrm{q}+}$ Ions with Uracil Phys. Rev. A 2005, 72, 052706/9.

(30) Bacchus-Montabonel, M.C., Tergiman, Y.S. Anisotropic Effect in the Charge Transfer of $\mathrm{C}^{\mathrm{q}+}$ Ions with Uracil Phys. Rev. A 2006, 74, 054702/4.

(31) Bacchus-Montabonel, M.C., Tergiman, Y.S. An Ab-Initio Study of Ion Induced Charge Transfer Dynamics in Collision of Carbon Ions with Thymine Phys. Chem. Chem. Phys. 2011, 13, 9761-9767.

(32) Salem, L. Electrons in Chemical Reactions: First principles; Wiley Interscience: New York, 1982.

(33) Bacchus-Montabonel, M.C.; Talbi, D.; Persico, M. Quantum Chemical Determination of the Rate Coefficients for Radiative Association of $\mathrm{CH}_{3}{ }^{+}$and $\mathrm{H}_{2} J$. Phys. B 2000, 33, 955-959.

(34) Bene, E.; Martínez, P.; Halász, G.J.; Vibók, Á.; Bacchus-Montabonel, M.C. Charge Transfer in Collisions of $\mathrm{C}^{2+}$ Carbon Ions with $\mathrm{CO}$ and $\mathrm{OH}$ Targets Phys. Rev. A 2009, 80, 012711/8.

(35) Bacchus-Montabonel, M.C.; Tergiman, Y.S. Radiation Damage on Biomolecular Systems: Dynamics of Ion Induced Collision Processes Comput. Theor. Chem. 2012, 990, 177-184.

(36) Bacchus-Montabonel, M.C. Anisotropy and Charge Effect in Collisions of Ions with Biomolecules Appl. Radiat. Isotopes 2014, 83, 95-99.

(37) MOLPRO (version 2012.1) is a package ab initio programs written by H.-J. Werner, P. J. Knowles, F. R. Manby, M. Schütz, P. Celani, G. Knizia, T. Korona, R. Lindh, A. Mitrushenkov, G. Rauhut et al.

(38) Nicklass, A.; Dolg, M.; Stoll, H.; Preuss, H. Ab Initio Energy-Adjusted Pseudopotentials for the Noble Gases Ne through Xe: Calculation of Atomic Dipole and Quadrupole Polarizabilities J. Chem. Phys. 1995, 102, 8942-8953. 


\section{Figure captions}

Figure 1. Calculated lowest unoccupied molecular orbitals (LUMOs) for uracil in the presence of a potassium cation.

Figure 2. Calculated lowest unoccupied molecular orbitals (LUMOs) for thymine in the presence of a potassium cation.

Figure 3. Negative ion TOF mass spectra showing $\mathrm{m} / \mathrm{z}=42$ formation for potassium-uracil at different laboratory collision energies.

\section{Table captions}

Table 1. Laboratory frame collision energies (in $\mathrm{eV}$ ) and their related centre-of-mass available energies (in $\mathrm{eV}$ ) for collisions of potassium atoms with uracil molecules. 
Figure 1. Calculated lowest unoccupied molecular orbitals (LUMOs) for uracil in the presence of a potassium cation in the perpendicular geometry. Energies in $\mathrm{eV}$.

\begin{tabular}{|c|c|c|}
\hline$(\mathrm{U}-\mathrm{H})^{-}$ & Uracil + K & Uracil (DEA) \\
\hline $\begin{array}{l}\pi_{1}^{*} \\
\pi_{2}^{*}\end{array}$ & 2.3 & 4.3 \\
\hline$\pi_{3} *$ & 10.7 & 9.8 \\
\hline$\sigma_{1}^{*}$ & 6.6 & 5.2 \\
\hline
\end{tabular}

Uracil (DEA) refers to uracil from Dissociative Electron Attachment; $\mathrm{K}^{+}$is schematically represented inside the pyrimidyne ring 
Figure 2. Calculated lowest unoccupied molecular orbitals (LUMOs) for thymine in the presence of a potassium cation in the perpendicular geometry. Energies in $\mathrm{eV}$.

\begin{tabular}{|l|c|c|}
\hline$(\mathrm{T}-\mathrm{H})^{-}$ & Thymine $+\mathrm{K}$ & Thymine (DEA) \\
\hline$\pi_{1}{ }^{*}$ & 2.3 & \\
\hline$\pi_{2}^{*}$ & & \\
\hline$\pi_{3}^{*}$ & & \\
\hline & & \\
\hline
\end{tabular}

Thymine (DEA) refers to thymine from Dissociative Electron Attachment; $\mathrm{K}^{+}$is schematically represented inside the pyrimidyne ring 
Figure 3. Negative ion TOF mass spectra showing $\mathrm{m} / \mathrm{z}=42$ formation for potassium-uracil at different laboratory collision energies.

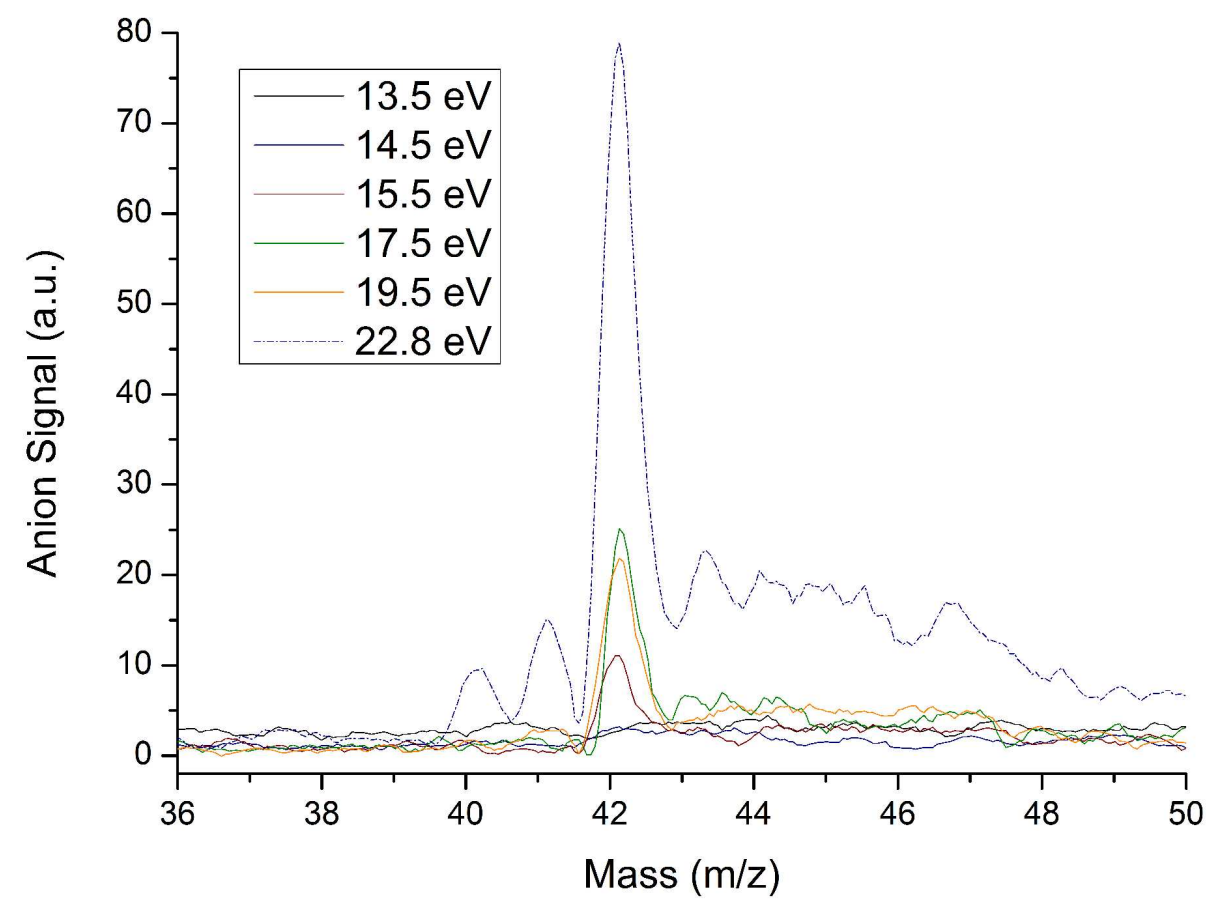


Table 1. Laboratory frame collision energies (in $\mathrm{eV}$ ) and their related centre-of-mass available energies (in $\mathrm{eV}$ ) for collisions of potassium atoms with uracil molecules.

\begin{tabular}{cc}
\hline Laboratory frame energy $(\mathrm{eV})$ & Available energy $(\mathrm{eV})$ \\
\hline \hline 13.5 & 4.6 \\
14.5 & 5.2 \\
15.5 & 5.9 \\
17.5 & 7.2 \\
19.5 & 8.5 \\
22.8 & 10.7 \\
\hline \hline
\end{tabular}


Table of Contents graphic

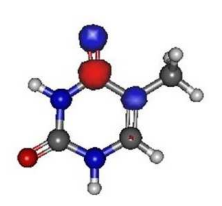

